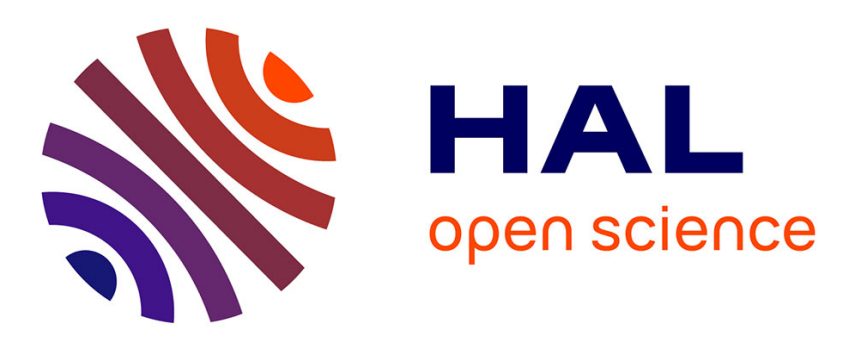

\title{
Micro-rheology of dense particulate flows: Application to immersed avalanches
}

Vincent Topin, Frédéric Dubois, Yann Monerie, Frédéric Perales, Anthony Wachs

\section{- To cite this version:}

Vincent Topin, Frédéric Dubois, Yann Monerie, Frédéric Perales, Anthony Wachs. Micro-rheology of dense particulate flows: Application to immersed avalanches. Journal of Non-Newtonian Fluid Mechanics, 2011, 166 (1-2), pp.63-72. 10.1016/j.jnnfm.2010.10.006 . hal-00806645

\section{HAL Id: hal-00806645 https://hal.science/hal-00806645}

Submitted on 26 Apr 2019

HAL is a multi-disciplinary open access archive for the deposit and dissemination of scientific research documents, whether they are published or not. The documents may come from teaching and research institutions in France or abroad, or from public or private research centers.
L'archive ouverte pluridisciplinaire HAL, est destinée au dépôt et à la diffusion de documents scientifiques de niveau recherche, publiés ou non, émanant des établissements d'enseignement et de recherche français ou étrangers, des laboratoires publics ou privés. 


\title{
Micro-rheology of dense particulate flows: Application to immersed avalanches
}

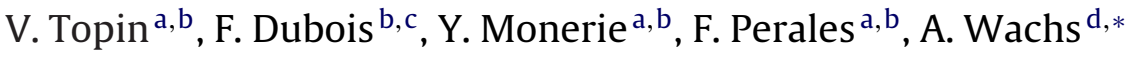 \\ a IRSN, DPAM, CE Cadarache, BP3 - 13115 St Paul-Lez-Durance Cedex, France \\ b MIST Laboratory, CNRS - IRSN - Université Montpellier 2, France

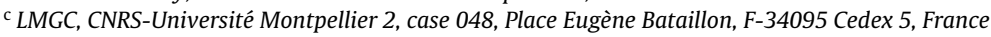 \\ ${ }^{\mathrm{d}}$ IFP Energies nouvelles, Fluid Mechanics Department, 1 \&4 avenue de Bois Préau, 92852 Rueil Malmaison Cedex, France
}

\begin{abstract}
A B S T R A C T
We rely here on a non-smooth contact dynamics (NSCD) approach to treat particle collisions in a direct numerical simulation of a dense particulate flow. Interactions between particles are considered by a non-smooth formulation of particle dynamics at the microscopic scale, which enables one to straightforwardly implement complex contact laws. The hydrodynamic coupling is achieved by a distributed Lagrange multiplier/fictitious domain (DLM/FD) method. As a preliminary step, the relevance of our NSCD-DLM/FD method is assessed by comparing results of 2D sedimentation simulations with those obtained with a usual molecular dynamics collision model. Then, we use it to investigate how a fully immersed granular packing collapses depending on its initial particle volume fraction, providing clues on the micro-rheology of dense particulate flows.
\end{abstract}

Keywords:

Dense particulate flows

Contact rheology

Immersed avalanches

Distributed Lagrange multiplier/fictitious

domain

Non-smooth contact dynamics

\section{Introduction}

The field of granular multi-phase flows has received over the past decades a very deep attention from the scientific community for assorted reasons, one of them being the broad range of both industrial and natural processes in which the momentum transfer between the discrete and continuous phases affects significantly the dynamics of the flow as a whole. For example, slurry transport in pipelines, dispersion of fuel fragments into coolant water through a hypothetic nuclear accident, sedimentation in water treatment, fluidization in catalyst reactors or debris flow can be considered as multi-phase systems presenting an evolutive texture characterized by the coexistence between a granular phase and a saturating liquid (see for instance the excellent review book of Chhabra [1]). Though some macroscopic models derived from experimental observations and theoretical works are able to reproduce simple mechanical behaviors [2-6], the physical mechanisms which occur at the particle scale in more complex phenomena involving collections of particles (big size hydrodynamic instabilities, formation of clusters, collapse, transport, etc.) have not been yet either well identified or accounted for. In particular, when the solid phase reaches a high volume fraction, the strong heterogeneity in terms of contact forces

\footnotetext{
* Corresponding author.

E-mail addresses: anthony.wachs@ifp.fr, anthony.wachs@ifpenergiesnouvelles.fr (A. Wachs).
}

between particles [7] and the influence of hydrodynamic forces on the granular assembly cannot be easily integrated in a homogenization process based on simplifying assumptions and global averages. In classical fluid/solid flow macroscopic approaches, as in twophase flow or mixture models, the dispersed solid phase is often modeled by a set of continuous conservation equations. In standard two-phase flow models, the momentum transfer between the particles and the suspending fluid relies on an additional source term in the continuous momentum equations of both phases, whose mathematical form is often complex and differs in the literature $[2,3,8]$. In mixture models, like the one suggested by Philipps et al. [4] in which shear-induced migration and particles collisions are considered in an average way, the presence of the particles is tracked by a continuous concentration equation and the macroscopic properties of the mixture in terms of viscosity rely on a Krieger-like relation. Though these models do provide non-homogeneous particles concentration in the flow, local variations at the scale of the particle have been somehow squeezed in the homogenization process from the micro to the macro-scale. In the specific case of immersed avalanche as we shall see later in this paper, modeling the dispersed phase as a pseudo-continuous one like in a two-fluid flow or mixture model would yield an instantaneous onset of the avalanche due to the density difference between the two phases. We will show later on that the granular texture leads to a totally different behavior, involving a significant initial delay before the avalanche triggers.

From a granular viewpoint, the discrete numerical models such as molecular dynamics (MD) and non-smooth contact dynamics 
(NSCD) are based on a microscopic description of the system at the particle scale, allowing to consider without difficulty complex textures that might involve polydisperse size distribution, specific contact laws (friction, cohesion, capillarity) or various shapes for the particles [9-13]. In the presence of a liquid phase, however, an adequate description at the microscopic scale requires in addition the coupling with a fluid method (lattice Boltzmann, finite element, finite volume) to properly account for the effect of hydrodynamic forces on the granular phase. In this context, direct numerical simulations (DNS) provide an adequate framework to simulate particulate flows at high concentration. The major issue stems from the fact that the space occupied by the fluid is constantly evolving as the particles move. In the fixed-mesh method case, the two main approaches generally used to overcome this issue are the immersed boundary (IB) method [14] and the distributed Lagrange multiplier/fictitious domain (DLM/FD) method [15]. The former implies to add appropriate boundary forces directly calculated from the immersed frontiers between the fluid and the particles, and the latter to extend the domain occupied by the fluid to the bulk of the particles (the fictitious domain) and to impose a rigid body motion constraint thanks to Lagrange multipliers (see more details in Section 2).

In this paper, we rely on our DNS tool of NSCD-DLM/FD type to investigate immersed avalanches of dense granular packings in a Newtonian fluid. The model presented here is an extension of our previous work [16] in which we replace the MD granular solver by a NSCD based one. The numerical developments have been performed in the framework of the PeliGRIFF platform [17] which has been coupled with LMGC90 [18], a NSCD code dedicated to contact problems (the resulting software has been named ApoloGRIFF by IRSN). The main asset of LMGC90 is its large range of contact laws already implemented and its ability to consider more complex surface interactions as cohesion, wear, etc. Furthermore, the NSCD solver treats frictional contact without neither regularization nor penalization, which presents a simplification compared to MD solvers. Hence, the recent extension of PeliGRIFF to MPI capabilities in addition with the coupling with LMGC90 makes our NSCD-DLM/FD code a powerful tool able to simulate large 2D and $3 \mathrm{D}$ collections of particles immersed in a fluid and interacting each other throughout specific contact laws. The main objective here is to evidence how, from a detailed description of the rheology at the microscopic scale, such a model is able to (i) capture complex physical mechanisms observed experimentally and (ii) monitor and evaluate local variables governing the global behavior. It is however fair to acknowledge that it is likely that results similar to the ones presented in Section 3 for immersed avalanches computed with our NSCD-DLM/FD code could have been obtained with a MDDLM/FD one, though we have not attempted it. Hence, though our NSCD-DLM/FD coupling is an original contribution to the literature, our primary interest here pertains to the physical understanding we gain from such a DNS survey in terms of rheological behavior.

In the following, we first describe in some details the numerical model. We recall how the DLM/FD variational formulation is derived in the general case, i.e., without any assumption on the particles collision model, and detail the main principle of the NSCD approach as a granular solver in the case of rigid bodies. We compare briefly in Section 2.3 numerical results obtained with our NSCD-DLM/FD code with data available in the literature on a basic sedimentation test. In Section 3, we employ our numerical model to investigate the collapse of immersed particles column with a focus on the role of initial compacity on the destabilization process. We briefly present a short review of the main experimental and numerical contributions on granular flows in the literature and then propose a 2D parametric study as a function of initial particle volume fraction and Reynolds number. We finally conclude with remarks about the specificity of dense immersed granular flows and extensions of this work in Section 4.

\section{Numerical model}

\subsection{Distributed Lagrange multiplier/fictitious domain method}

In this section, we present how the DLM/FD global variational formulation for the fluid/particles mixture is derived. Though this derivation has already been detailed elsewhere in the literature $[15,16]$, we shortly recall the various steps that lead to the final form of the combined momentum equation. Indeed, we start from the conservation equations of each phase separately to highlight the fact that the formulation is clearly independent from the collision model. This property enables us to employ our new NSCD approach without altering the DLM/FD hydrodynamic coupling.

\subsubsection{Governing equations}

Let us consider a bounded domain $\Omega$ and $\Gamma$ its boundary. We suppose that $\Omega$ is filled by $N_{p}$ particles $P_{i}$ where $i \in\left(1, N_{p}\right)$. We note $P$ the total bulk of the particles and $\delta P$ their frontiers. The motion of a Newtonian and incompressible fluid in a particle-filled space is described by the following equations:

$$
\begin{aligned}
& \rho_{f} \frac{d \boldsymbol{u}_{f}}{d t}=\nabla \cdot \sigma_{f}+\rho_{f} \boldsymbol{f}_{f} \quad \text { on } \Omega \backslash P \\
& \nabla \cdot \boldsymbol{u}_{f}=0 \text { on } \Omega \backslash P \\
& \boldsymbol{u}_{f}=\boldsymbol{u}_{f, \Gamma} \text { on } \Gamma \\
& \boldsymbol{u}_{f}=\boldsymbol{u}_{s} \text { on } \delta P
\end{aligned}
$$

where $\rho_{f}, \boldsymbol{u}_{f}, \boldsymbol{f}_{f}$ and $\boldsymbol{\sigma}_{f}$ are the density, velocity, body force density and stress tensor of the fluid, respectively. The stress tensor can be further decomposed as $\boldsymbol{\sigma}_{f}=-p \mathbf{1}+\boldsymbol{\tau}_{f}$, involving the pressure $p$ and the deviatoric component $\boldsymbol{\tau}_{f}$. $\boldsymbol{u}_{s}$ stands for the particles velocity. Eqs. (1) and (2) correspond to the Navier-Stokes equations, (3) is a Dirichlet condition where a given velocity $\boldsymbol{u}_{f, \Gamma}$ is imposed on $\Gamma$ and (4) represents a no-slip condition imposed at the interface $\delta P$ between fluid and particles.

In the same way, the motion equation of a set of particles immersed in a fluid can be written as:

$\rho_{s} \frac{d \boldsymbol{u}_{s}}{d t}=\nabla \cdot \boldsymbol{\sigma}_{s}+\rho_{s} \boldsymbol{f}_{s}$ in $P$,

where $\rho_{s}, \boldsymbol{f}_{s}$ and $\boldsymbol{\sigma}_{s}$ are the density, body force density and stress tensor of the solid particles, respectively. The boundary conditions at the interface in terms of normal stress implies that $\sigma_{s}$ is the sum of a fluid contribution $\sigma_{f}$ and a contact contribution $\sigma_{c}$ such as:

$\boldsymbol{\sigma}_{s} \boldsymbol{n}_{s}=\boldsymbol{\sigma}_{f} \boldsymbol{n}_{s}+\boldsymbol{\sigma}_{c} \boldsymbol{n}_{s}$ on $\delta P$,

where $\boldsymbol{n}_{s}$ denotes the normal direction.

The weak formulation of the fluid/particles problem is written, in dimension $d$, as:

$$
\text { Find }\left(\boldsymbol{u}_{f}, \boldsymbol{u}_{s}\right) \in \vartheta(\Omega) \text { such that: }
$$

$$
\begin{aligned}
& \int_{\Omega \backslash P}(1) \cdot v_{f} d \Omega+\int_{P}(5) \cdot v_{s} d \Omega=0, \quad \forall\left(v_{f}, v_{s}\right) \in \vartheta_{o}(\Omega) \\
& \int_{\Omega \backslash P}(2) \cdot q d \Omega=0, \quad \forall q \in \mathcal{L}_{0}^{2}(\Omega),
\end{aligned}
$$

where

$$
\begin{aligned}
& \vartheta(\Omega)=\left\{\left(\boldsymbol{v}_{f}, \boldsymbol{v}_{s}\right) / \boldsymbol{v}_{f} \in \mathcal{H}^{1}(\Omega \backslash P)^{d}, \quad \boldsymbol{v}_{s} \in \mathcal{H}^{1}(P)^{d}, \quad \boldsymbol{v}_{f}=\boldsymbol{v}_{s} \text { on } \delta P, \boldsymbol{v}_{f}=\boldsymbol{u}_{f, \Gamma} \text { on } \Gamma\right\}, \\
& \vartheta_{o}(\Omega)=\left\{\left(\boldsymbol{v}_{f}, \boldsymbol{v}_{s}\right) / \boldsymbol{v}_{f} \in \mathcal{H}^{1}(\Omega \backslash P)^{d},\right. \\
& \left.\boldsymbol{v}_{s} \in \mathcal{H}^{1}(P)^{d}, \quad \boldsymbol{v}_{f}=\boldsymbol{v}_{s} \text { on } \delta P, \quad \boldsymbol{v}_{f}=0 \text { on } \Gamma\right\} .
\end{aligned}
$$


As introduced by Glowinski et al. [15], the main idea of the fictitious domain method is to extend the problem defined on $\Omega \backslash P$ to the entire domain $\Omega$, and to force the solution to satisfy the condition $\boldsymbol{u}_{f}=\boldsymbol{u}_{s}$ in $P$. In other words, it requires to extend the condition (4) from $\delta P$ to $(\delta P+P)$ in formulation (7) and to relax the constraint $\boldsymbol{u}_{f}=\boldsymbol{u}_{s}$ in $P$ by introducing a Lagrange multiplier. Besides, we consider here rigid particles $\left(D\left(\boldsymbol{u}_{s}\right)=0\right)$, incompressible fluid $\left(\nabla \cdot \boldsymbol{u}_{f}=0\right)$ and only gravity body forces $\left(\boldsymbol{f}_{f}=\boldsymbol{f}_{s}=\boldsymbol{g}\right)$. We note that the particles velocity $\boldsymbol{u}_{s}$ can be written as the sum of the translational velocity $\boldsymbol{u}_{s}^{t} \in \mathbb{R}^{d}$ and angular velocity $\boldsymbol{\omega} \in \mathbb{R}^{\bar{d}}$ as $\boldsymbol{u}_{s}=\boldsymbol{u}_{s}^{t}+\boldsymbol{\omega} \times \boldsymbol{r}$, with $\boldsymbol{r} \in \mathbb{R}^{d}$ the position vector with respect to particles gravity center and $\bar{d}$ is the number of non-zero components of $\omega$. Finally, we obtain the following combined equations of motion for the extended domain:

Find $\boldsymbol{u}_{f} \in \mathcal{V}_{\Gamma}(\Omega), \boldsymbol{u}_{s}^{t} \in \mathbb{R}^{d}, \boldsymbol{\omega} \in \mathbb{R}^{\bar{d}}, \lambda \in \mathcal{H}^{1}(P)^{d}$ such that:

(a) Combined momentum equations

$$
\begin{aligned}
& \int_{\Omega} \rho_{f}\left(\frac{\partial \boldsymbol{u}_{f}}{\partial t}+\left(\boldsymbol{u}_{f} \cdot \nabla\right) \boldsymbol{u}_{f}-\boldsymbol{g}\right) \cdot \boldsymbol{v}_{f} d \Omega-\int_{\Omega} p \nabla \cdot \boldsymbol{v}_{f} d \Omega \\
& +\int_{\Omega} \boldsymbol{\tau}_{f}: \boldsymbol{D}\left(\boldsymbol{v}_{f}\right) d \Omega+\left(1-\frac{\rho_{f}}{\rho_{s}}\left(M\left(\frac{d \boldsymbol{u}_{s}^{t}}{d t}-\mathbf{g}\right) \cdot \boldsymbol{v}_{s}^{t}+\mathbf{1} d \boldsymbol{\omega} / d t \cdot \boldsymbol{\xi}\right)\right. \\
& \quad-\quad \boldsymbol{F}_{c j} \cdot \boldsymbol{v}_{s}^{t}-{ }_{j} \boldsymbol{F}_{c j} \cdot\left(\boldsymbol{\xi} \times \boldsymbol{R}_{j}\right)+\int_{P} \lambda \cdot\left(\boldsymbol{v}_{f}-\left(\boldsymbol{v}_{s}^{t}+\boldsymbol{\xi} \times \boldsymbol{r}\right)\right) d \Omega=0, \\
& \quad \forall v_{f} \in \mathcal{V}_{0}(\Omega), \boldsymbol{v}_{s}^{t} \in \mathbb{R}^{d}, \boldsymbol{\xi} \in \mathbb{R}^{\bar{d}},
\end{aligned}
$$

$\int_{P} \boldsymbol{\gamma} \cdot\left(\boldsymbol{u}_{f}-\left(\boldsymbol{u}_{s}^{t}+\boldsymbol{\omega} \times \boldsymbol{r}\right)\right) d \Omega=0, \quad \forall \boldsymbol{\gamma} \in \mathcal{H}^{1}(P)^{d}$

(b) Continuity equation

$-\int_{\Omega} q \nabla \cdot \boldsymbol{u}_{f} d \Omega=0, \quad \forall q \in \mathcal{L}_{0}^{2}(\Omega)$.

where $\lambda$ is the Lagrange multiplier field, $\left(\boldsymbol{v}_{f}, q, \boldsymbol{\gamma}, \boldsymbol{v}_{s}^{t}, \boldsymbol{\xi}\right)$ correspond to the test functions for $\left(\boldsymbol{u}_{f}, p, \boldsymbol{\lambda}, \boldsymbol{u}_{s}^{t}, \boldsymbol{\omega}\right), \boldsymbol{F}_{c j} \in \mathbb{R}^{d}$ the contact forces, $\boldsymbol{R}_{j} \in \mathbb{R}^{d}$ the vectors between particle gravity center and contact point, $M \in \mathbb{R}$ the particle mass and $\mathbf{1} \in \mathbb{R}^{\bar{d} \times \bar{d}}$ the particles inertia tensor. We have introduced in the equations above the two following functional spaces:

$$
\left.\mathcal{V}_{0}(\Omega)=v_{f} \in \mathcal{H}^{1}(\Omega)^{d} / v_{f}=0 \text { on } \Gamma\right\}
$$

$\mathcal{V}_{\Gamma}(\Omega)=\boldsymbol{v}_{f} \in \mathcal{H}^{1}(\Omega)^{d} / \boldsymbol{v}_{f}=\boldsymbol{u}_{f, \Gamma}$ on $\left.\Gamma\right\}$

It is fairly important to note that, up to this stage, no assumption on the way the contact forces $\boldsymbol{F}_{c j}$ are calculated, i.e., the type of granular solver, has been introduced. This property makes the DLM/FD hydrodynamic coupling independent of the collision model and enables us to implement the most appropriate one.

\subsubsection{Computational scheme}

Concerning the computational scheme, we employ the first order Marshuk-Yanenko operator-splitting algorithm for time integration of the problem (9)-(11)[15,16]. This method enables one to decouple the whole problem into a serie of sub-problems $i$ simpler to solve for which we implement optimized and efficient solution methods. At each iteration $n$, the whole problem is split into five steps, corresponding to the five following simpler problems [16]:

(1) Degenerated Stokes problem

(2) Transport problem

(3) Viscous problem

$\Rightarrow$ find $\boldsymbol{u}_{f}^{n+(i / 5)}$ from $\boldsymbol{u}_{f}^{n+(i-1 / 5)}, \quad i \in\{1,2,3\}$

(4) Granular problem (see Section 2.2)

$\Rightarrow$ find $\boldsymbol{u}_{s}^{t, n+4 / 5}, \boldsymbol{\omega}^{n+4 / 5}$ from $\boldsymbol{u}_{s}^{t, n}, \omega^{n}$

(5) Fictitious domain problem

$\Rightarrow$ find $\boldsymbol{u}_{f}^{n+1}, \boldsymbol{u}_{s}^{t, n+1}, \omega^{n+1}$ from $\boldsymbol{u}_{f}^{n+4 / 5}, \boldsymbol{u}_{s}^{t, n+4 / 5}, \omega^{n+4 / 5}$

At each time step, the splitting method leads to solve independently the Navier-Stokes equations (steps 1-3) and the granular problem (step 4); and to finally correct fluid and particles velocities together (step 5). In this paper, the additional contribution pertains to the choice of the granular solver. In fact, we use here for the first time with a DLM/FD method a non-smooth contact dynamics approach to simulate the granular phase. This extension allows to treat frictional contact interactions between particles without neither ad hoc regularization nor penalty method.

\subsection{Granular approach: non-smooth contact dynamics method}

The step 4 of our operator-splitting scheme (see Section 2.1.2) aims at calculating the new velocity of each particle independently from any fluid interactions, i.e., as if we were simulating a dry granular packing. Here we suggest a non-smooth contact dynamics (NSCD) approach, first introduced by Moreau and Jean $[19,20]$, which is a powerful alternative method to common molecular dynamics (MD) methods usually employed in granular simulations.

The fundamental difference between NSCD and MD lies in the treatment of small length and time scales involved in the dynamics of granular media. Schematically, the MD method is based on a description of particle interactions in terms of force laws, i.e., objective force-displacement relations, whereas the NSCD method consists in formulating kinematic constraints in terms of contact laws. The NSCD method does not involve small interpenetrations between particles as with a MD method, and the algorithm used is unconditionally stable due to an implicit discretization. In the present case, the asset of considering strictly rigid particles ensures the incompressibility of the surrounding fluid. Concerning the computational scheme, the implicit algorithm allows one to use the same step for the granular solver as for the fluid solver (while the MD solver requires several sub-iterations to ensure small interpenetrations between particles). Although bodies with complex interaction laws (cohesion, wet contact, etc.) can be easily treated in the framework of the NSCD method $[12,21,22]$, we focus here on the case of rigid particles interacting with each other according to an unilateral frictional contact law (Signorini-Coulomb).

In this context, the method can be described in three steps:

(1) The free velocity of all particles is calculated without contact forces from the equation of dynamics expressed in terms of velocity jumps.

(2) All kinematic constraints implied by enduring contacts are simultaneously considered in order to determine the relative velocities and forces at the contact scale.

(3) The particles velocity and position are updated in the equation of dynamics taking into account the contribution of contact forces calculated above at step 2 .

The NSCD method is thus based on a mechanical description of the system at two different scales, a global and a contact scale, the transition from one scale to the other one being possible via a mapping which contains the geometry of each contact. 


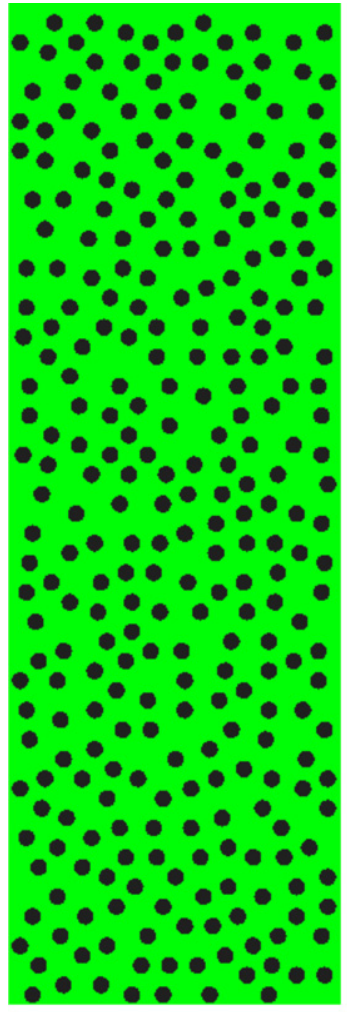

$\mathrm{t}_{0}$

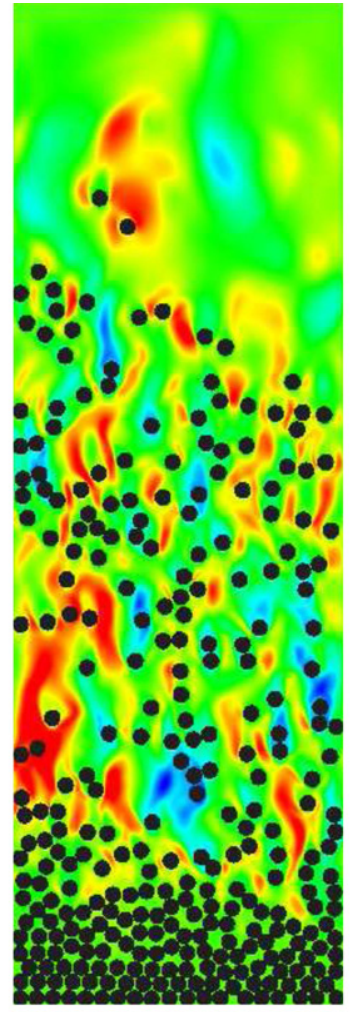

$\mathrm{t}_{1}$

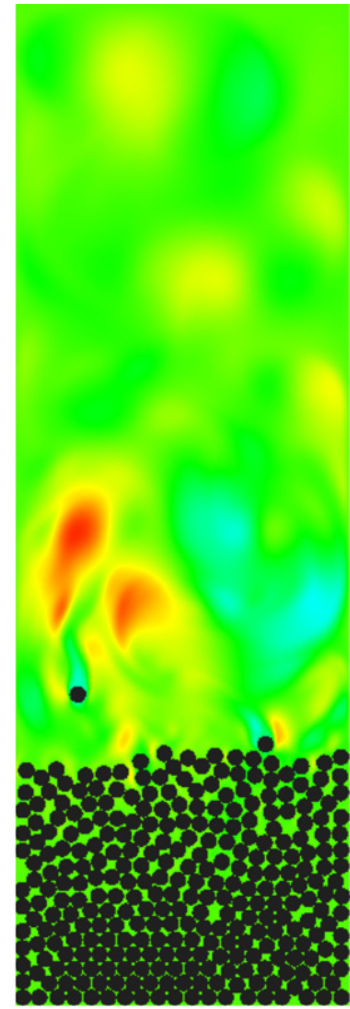

$t_{2}$

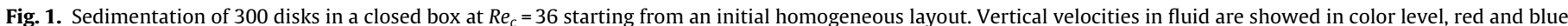

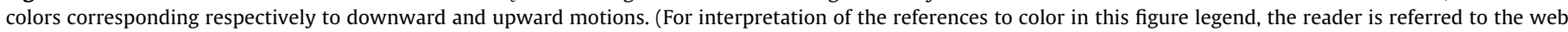
version of the article.)

\subsubsection{Global scale: the dynamics equation}

The standard dynamics equation (see Eq. (5)) for each body can be written in a discrete form such that:

$\boldsymbol{M} . d \dot{\boldsymbol{x}}=\boldsymbol{F}(\boldsymbol{x}, \dot{\boldsymbol{x}}, t)+d \boldsymbol{c}$

$\boldsymbol{x}(t)=\boldsymbol{x}\left(t_{0}\right)+\int_{t_{0}}^{t} \dot{\boldsymbol{x}} d t$

where $\boldsymbol{M}$ is the mass matrix, $\boldsymbol{x}$ is the discrete displacement, $\dot{\boldsymbol{x}}$ the discrete velocity associated to $\boldsymbol{u}_{s}, d \dot{\boldsymbol{x}}$ the differential measure of the velocity, $d \boldsymbol{c}$ the differential measure of interaction impulse and $\boldsymbol{F}(\boldsymbol{x}, \dot{\boldsymbol{x}}, t)$ corresponds to the internal and external forces, i.e., only the gravity forces for rigid bodies $[23,24]$.

When time discretization is performed, an elementary subinterval $] t_{i} ; t_{i+1}$ ] of length $h$ is considered. Using an implicit time integration scheme $(\theta$-method), we obtain the following discrete form (index $i$ stands here for time step $t_{i}$ ):

$\boldsymbol{M}\left(\dot{\boldsymbol{x}}_{i+1}-\dot{\boldsymbol{x}}_{i}\right)=h\left(\theta \boldsymbol{F}\left(\boldsymbol{x}_{i+1}, \dot{\boldsymbol{x}}_{i+1}, t_{i+1}\right)+(1-\theta) \boldsymbol{F}\left(\boldsymbol{x}_{i}, \dot{\boldsymbol{x}}_{i}, t_{i}\right)\right)+\boldsymbol{c}$
$\boldsymbol{x}_{i+1}=\boldsymbol{x}_{i}+h\left(\theta \dot{\boldsymbol{x}}_{i+1}+(1-\theta) \dot{\boldsymbol{x}}_{i}\right)$.

In the case of rigid bodies, the variable $\boldsymbol{x}$ corresponds to the gravity center coordinates vector of the particles and $\boldsymbol{F}(\boldsymbol{x}, \dot{\boldsymbol{x}}, t)=$ $\boldsymbol{G}(t)$, where $\boldsymbol{G}$ contains volume forces.

Hence, the problem at the global scale can be written as:

$\dot{\boldsymbol{x}}_{i+1}=\dot{\boldsymbol{x}}_{\text {free }}+\boldsymbol{w c}$

$\dot{\boldsymbol{x}}_{\text {free }}=\dot{\boldsymbol{x}}_{i}+\boldsymbol{w h}\left(\theta \boldsymbol{G}_{i+1}+(1-\theta) \boldsymbol{G}_{i}\right)$

$\boldsymbol{x}_{i+1}=\boldsymbol{x}_{i}+h\left(\theta \boldsymbol{x}_{i+1}+(1-\theta) \boldsymbol{x}_{i}\right)$

with $\boldsymbol{w}=\boldsymbol{M}^{-1}$

\subsubsection{Contact scale: kinematic relations}

At the contact scale, a mapping $H$ is introduced between the bulk variables $(\dot{\boldsymbol{x}}, \boldsymbol{c})$ at the global scale and the contact variables, i.e., the relative velocity $\dot{\boldsymbol{X}}$ and impulse $\boldsymbol{C}$. Considering each contact $\alpha$ between two objects indexed $k$ and $l$, the dynamics problem can be written in terms of contact variables in the following way:

$\dot{\boldsymbol{X}}^{\alpha}=\dot{\boldsymbol{X}}_{f r e e}^{\alpha}+\boldsymbol{W}^{\alpha \alpha} \boldsymbol{C}^{\alpha}+\underset{\beta \neq \alpha}{\boldsymbol{W}^{\alpha \beta} \boldsymbol{C}^{\beta}}$

where $\dot{\boldsymbol{X}}_{\text {free }}^{\alpha}$ is the free relative velocity obtained without contact force, $\beta$ accounts for the contributions of all other contacts $\neq \alpha$ and $\boldsymbol{W}^{\alpha \beta}=H_{k}^{T \alpha} \boldsymbol{w} H_{k}^{\beta}-H_{l}^{T \alpha} \boldsymbol{w} H_{l}^{\beta}$.

Eq. (19) defines a system of linear equations between the contact variables at each contact point. We point out that, in the context of mutual exclusion and dry friction between particles, the solution $\left(\dot{\boldsymbol{X}}^{\alpha}, \boldsymbol{C}^{\alpha}\right)$ should also verify a non-smooth frictional contact law (Signorini-Coulomb in the following). To solve all contacts, the NSCD method relies on a non-linear gauss seidel (NLGS) algorithm. At step 1, for each contact problem $\alpha$, we solve Eq. (19) with all other impulses $\beta$ kept constant. At step 2, we update impulses in all equations depending on the $\alpha$ impulse calculated at step 1 . We iterate step 1-step 2 process until a given convergence criterion is fulfilled. Due to the implicit time integration scheme inherent to the NSCD method, the solution is unconditionally stable. The particles position is then updated from the calculated particles velocity before a new detection of contacts between particles is performed.

\subsection{Example: $2 D$ sedimentation tests}

In this section, we briefly compare results obtained with our NSCD-DLM/FD model for a 2D sedimentation test to those obtained 


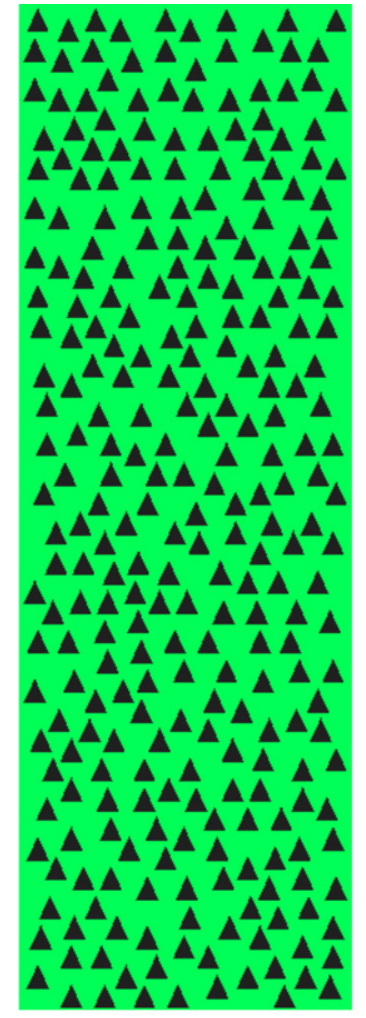

$\mathrm{t}_{0}$

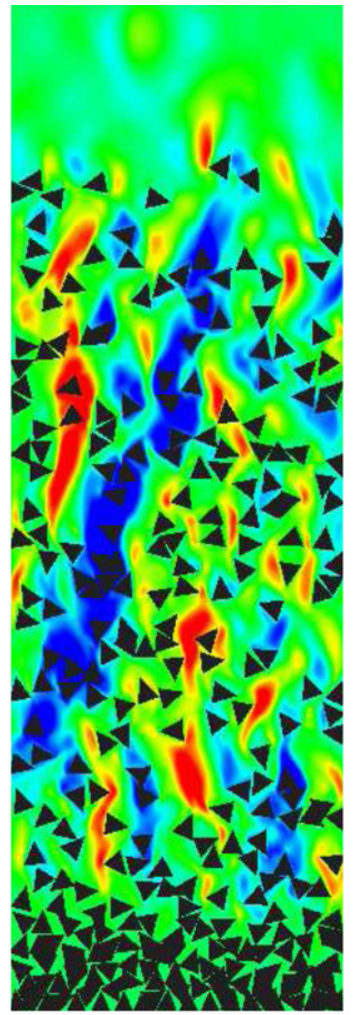

$\mathrm{t}_{1}$

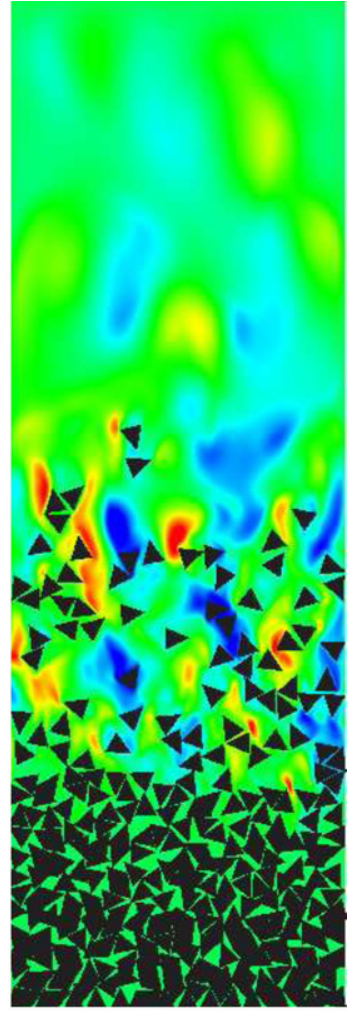

$\mathrm{t}_{2}$

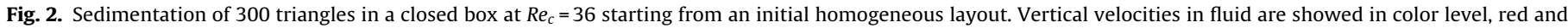

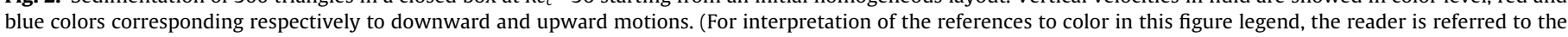
web version of the article.)

in [16] with a MD collision model implemented in the PeliGRIFF platform. We generated two granular samples composed respectively of 300 circular and 300 triangular particles distributed randomly in a box. The samples are chosen monodisperse with a diameter $D$ for the disks and a circumcircle diameter $D_{T}=1.5 D$ for the triangles resulting in both cases in a particle volume fraction $\phi=0.2$ in the box of dimensions $20 D \times 60 D$. We set the density ratio $\rho_{r}=\rho_{s} / \rho_{f}$ between particles and fluid to 1.5 , and the fluid viscosity $\eta$ depends on an imposed characteristic Reynolds number $R e_{c}=36$, $R e_{c}$ being calculated as:

$\operatorname{Re}_{c}=\frac{\rho_{f} u_{c} D}{\eta}$

where $u_{c}$ is a characteristic velocity given by the following Eq. [16]:

$u_{c}=\overline{\frac{\pi D}{2}\left(\rho_{r}-1\right) g(1-\phi)^{5}}$.

In all the simulations presented in this paper, the mesh has a constant size $\Delta l=D / 16$. In the present case, the mesh comprises 614,400 elements.

Figs. 1 and 2 show the evolution of the fluid/particles mixtures together with the vertical velocity of the fluid. We note that in both cases significant recirculations occur at low Reynolds number (laminar regime). In fact, though the mean motion of the particles is downward, many particles temporally move up. We plot in Fig. 3(a) the mean vertical velocity $\left\langle u_{y}\right\rangle$ in both cases as a function of time $t$. By convenience, $\left\langle u_{y}\right\rangle$ and $t$ are respectively non-dimensionalized by $u_{c}$ and $t_{c}=D / u_{c}$. In the same way, Fig. 3 (b) presents the evolution of the mean vertical position $\langle y\rangle$ of the particles scaled between 0 and 1 as a function of $t / t_{c}$.
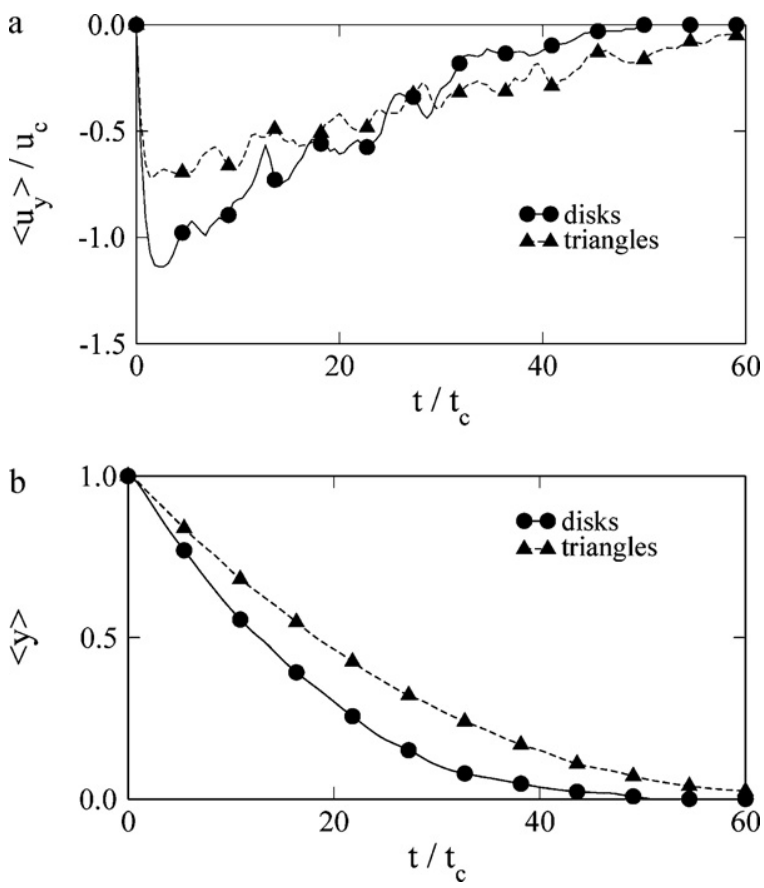

Fig. 3. Sedimentation of 300 particles (circular or triangular shape) in a closed box: (a) mean vertical velocity and (b) mean vertical position, plotted as a function of time. 

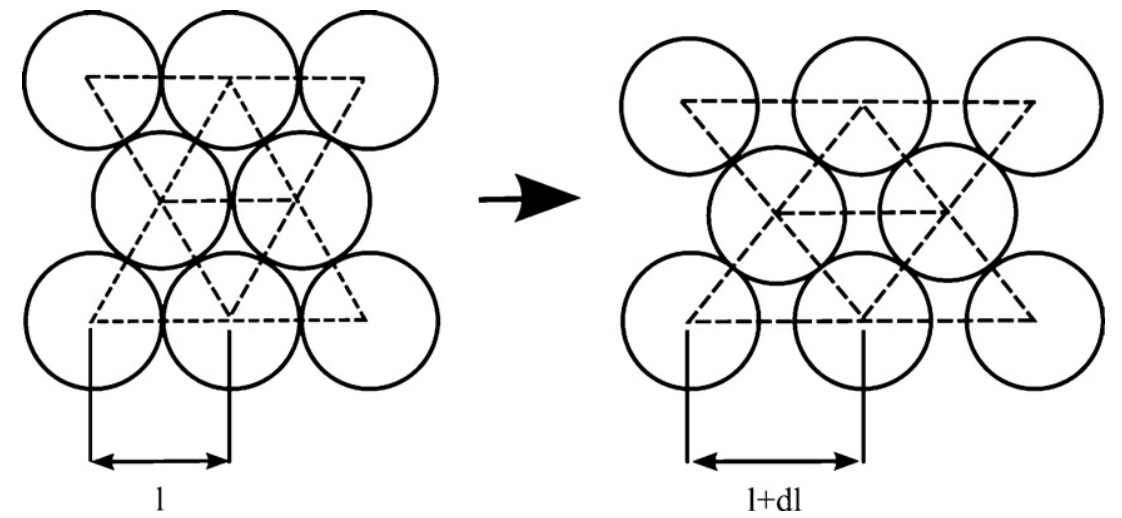

Fig. 4. Scheme representing the geometrical procedure used to vary the granular compacity in 2D.

It is worth pointing out that results are similar with those obtained in the same configurations with a MD model in [16]. In fact, the total sedimentation time of triangles is approximately $10 \%$ larger than the one of disks, due to the particle shape and the corresponding higher drag coefficient. Our goal here is not to analyze in detail the differences between disks and triangles (this has already been carefully achieved in [16]), but to evidence that, at least, the use of a NSCD method to tackle the multi-body collisions does not alter the satisfactory properties of the DLM/FD coupling. We admit that, in this particular sedimentation test we retained as a kind of validation test, the dynamics of the flow is not controlled by the multi-body contacts but more likely by the hydrodynamic interaction. However, the true scarcity of published results on the DNS of a suspension flow in which contacts are treated by either a MD or a NSCD method has motivated (almost constrained) our choice, the only work we are aware of being [16]. Based on this limited validation test, we claim that the nonsmooth contact dynamics approach is a well suited collision model to simulate dense fluid/particles mixtures which imply successive multi-contact configurations. As the reader will see in next section, our ability to simulate established experimental results will give additional credit to our numerical strategy.

\section{Application to immersed granular avalanches: fluid influence on the initiation of gravitational instabilities}

In this section, we rely on our NSCD-DLM/FD method to study numerically how the texture of a dense immersed granular packing influences the initiation of an avalanche. The understanding of immersed granular avalanches is a fundamental issue in many industrial applications and geophysical phenomena. For examples, landslides [25], debris flows [26] and submarine granular flows $[27,28]$ are natural events involving the mixture of a liquid (water) and a granular material composed in most cases of mud, sand, gravels or rocks. In the same way, many pharmaceutical or food processes imply the transport of powders or aggregates immersed in a fluid which plays the role of a lubricant or a binder [29]. In all cases, the presence of an interstitial liquid phase between the particles has profound effects on the collapse and the dynamics of dense assemblies of grains. In the dry case, i.e., when the interstitial fluid between particles does not play any role, many studies based on experiments [30-32] and discrete numerical simulations [32-35] have shown that the rheology of dense granular flows is only governed by friction and collisions between particles. On the opposite, in the presence of a fluid at the saturated state, gradients of fluid pressure develop inducing additional forces on the granular skeleton which can significantly enhance or reduce the motion of the packing as a whole [25]. In fact, the influence of the interstitial fluid on granular flows has been studied essentially experimentally in different configurations (inclined planes, rotating tumblers, etc.) in the past years $[28,36,37]$. It has been shown that a constitutive law obtained in the dry case can hold for immersed flows if a viscous time scale is introduced [28]. More recently, an experimental study inspired by Iverson et al. [25] demonstrated that the initiation and the dynamics of an immersed granular avalanche depend strongly on the initial particle volume fraction [38]. By analyzing how a layer of particles fully immersed in a liquid starts to flow when suddenly inclined from horizontal, the authors have shown that the flow initiation is dramatically delayed by a slight compaction. In this context, they proposed a model based on an interpretation of the coupling between the dilatant behavior of the granular material and the pore pressure developing in the interstitial fluid which is able to capture the main experimental features.

Simulations presented in this section are inspired by this last experimental result. We investigate numerically fully immersed granular collapses focusing on the role of initial particle volume fraction and Reynolds number. Our goal is to show how a detailed description of the rheology at the particle scale proves efficient to capture and follow the physical mechanisms which govern the complex triggering process observed experimentally at the scale of a large assembly of grains. In this way, we deliberately choose a geometry in which the collapse direction is not imposed as opposed to the experimental studies performed on an inclined plane.

\subsection{Study parameters}

We consider 2D mono-disperse samples composed of disks with a diameter $D$ and relative density $\rho_{r}=\rho_{s} / \rho_{f}=1.5$. It is worth mentioning that the objective of these $2 \mathrm{D}$ numerical simulations is not to fit the experimental data but to highlight the same physical phenomenon in a slightly different configuration. The choice of a 2D geometry has the advantage to require a cheaper computational effort than a 3D one, making it feasible to simulate very large systems with an important number of nodes for a reasonable computing time. By convenience, all problem variables are nondimensionalized: we use $l_{c}=D$ for length, $u_{c}=\overline{g D}$ for velocity and $t_{c}=l_{c} / u_{c}$ for time. The granular packings are $50 l_{c}$ height and $25 l_{c}$ wide which corresponds to approximatively between 1250 and 1450 particles located on a triangular lattice. The initial compacity $\phi$ of the various samples varies in the range [0.785;0.906], by stretching geometrically the underlying triangular network, as shown in Fig. 4. Actually, the densest sample corresponds to a underlying network composed of isosceles triangles $(\phi=\pi /(2 \sqrt{3}))$ whereas in the loosest case the particles are initially arranged on a rectangular grid $(\phi=\pi / 4)$. The granular packings are then immersed in a box of dimensions $250 l_{c} \times 100 l_{c}$ containing a fluid whose viscosity $\eta$ varies with the Reynolds number $R e_{c}=\rho_{f} u_{c} l_{c} / \eta$ such that $R e_{c}$ takes the following successive values: $89,8.9$, and 0.89 . For each 

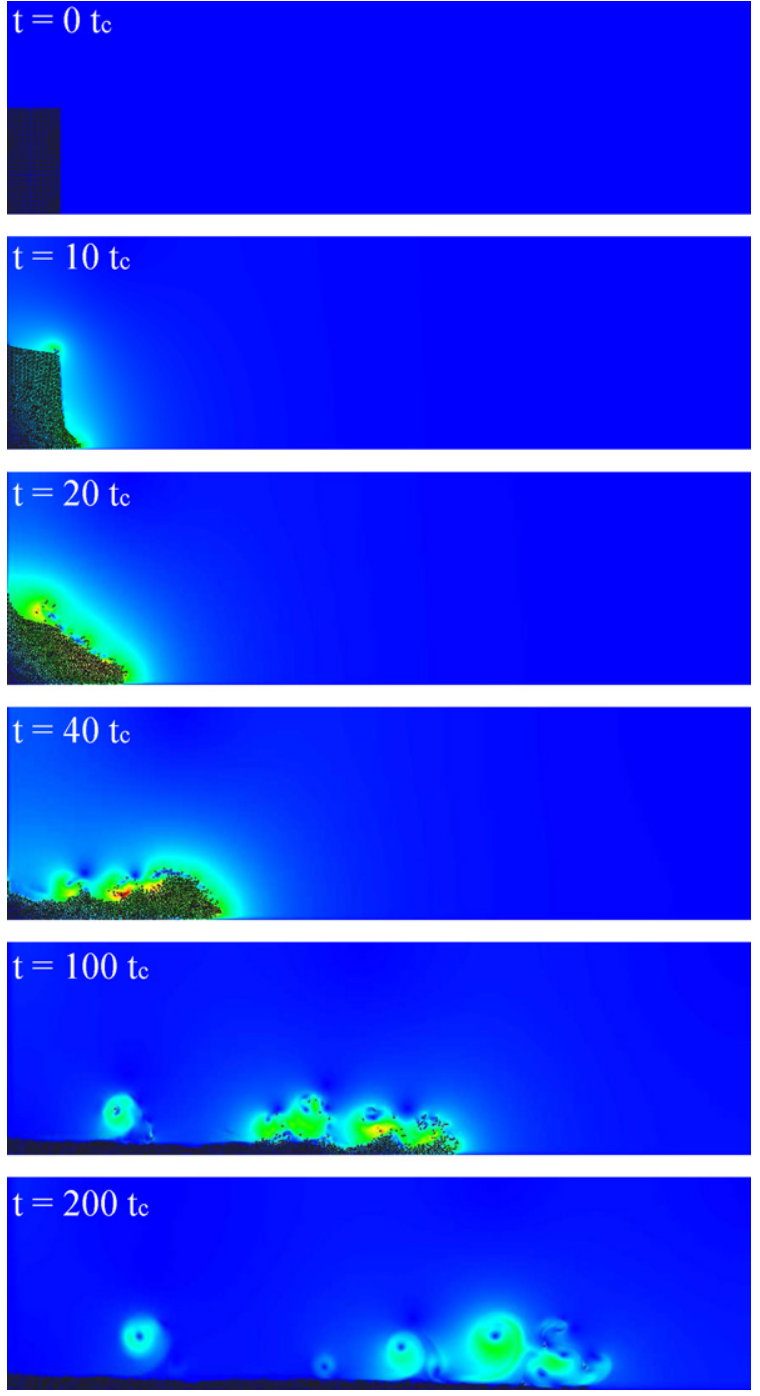

Fig. 5. Time sequence of images taken from the simulation of the immersed avalanche of a loose granular assembly $(\phi=0.785)$. The fluid velocities are shown in color levels. (For interpretation of the references to color in this figure legend, the reader is referred to the web version of the article.)

simulation, the fluid is solved on a mesh containing about 700,000 elements. A no-slip condition $\left(\boldsymbol{u}_{f}=0\right)$ is set on the left and bottom boundaries of the domain whereas the right and top ones are free. In order to compare immersed collapses with their dry counterpart, all simulations described above are also performed without fluid. In both cases, dry or saturated, interactions between particles follow a Signorini-Coulomb contact law with a friction coefficient equal to 0.3 and no shock restitution.

\subsection{Flow dynamics}

Figs. 5 and 6 show a time sequence of images taken from the simulations performed respectively for the loosest $(\phi=0.785)$ and the densest $(\phi=0.906)$ granular packings in a saturated case at $R e_{c}=89$. As already observed in the dry case [34], we note that the loosest packing seems to collapse in three phases: (1) a free-fall regime, where the top falls vertically and the particles localized at the bottom are ejected horizontally by the fluid; (2) a heap regime, where the upper particles move along an inclined stationary deposit; (3) a horizontal regime where the particles move essentially horizontally. In this last regime, fluid recirculations are observed leading to the formation of waves at the surface of the granular flow, as if the
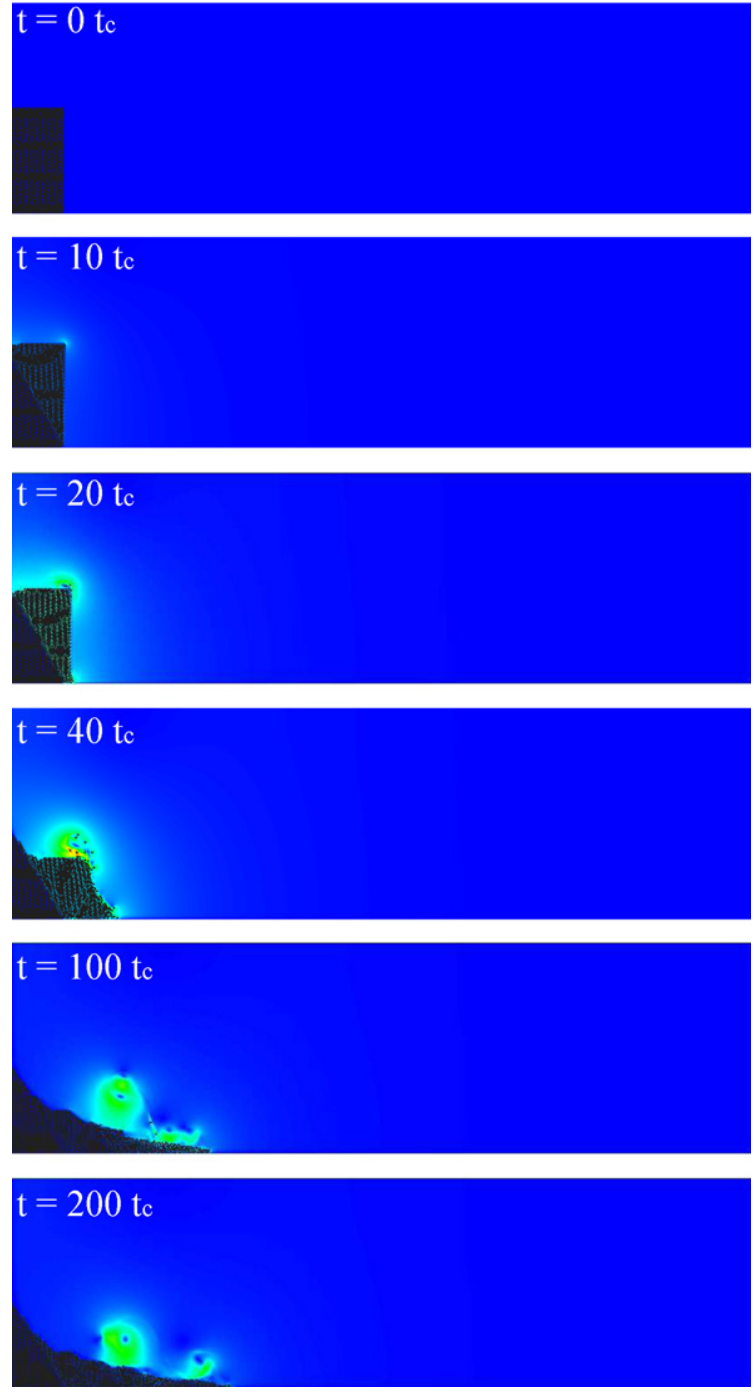

Fig. 6. Time sequence of images taken from the simulation of the immersed avalanche of a dense granular assembly $(\phi=0.906)$. The fluid velocities are shown in color levels. (For interpretation of the references to color in this figure legend, the reader is referred to the web version of the article.)

solid phase behaves like a second densest fluid [39]. To emphasize this aspect, we performed the same simulation in a smallest box with a no-slip condition on all boundaries (see Fig. 7). As expected the behavior is the same as previously till the granular flow impacts on the right wall. After the impact, the particles fall down under the gravity and start to move in the opposite direction generating a breaking wave which is usually observed in liquid sloshing tests [40]. On the opposite, the dense packing presents a collapse dynamics which differs significantly from the loose case (see Fig. 6). Indeed, the densest packing is sliced into two parts, the upper part sliding on a lower quiescent part along a preferred direction forming an angle of $45^{\circ}$ with the horizontal plane. We note that the dense avalanche is slower leading thus to a final spreading length which is about half that of the loosest one. It can be observed finally that no wave occurs in this case.

\subsection{Destabilization process}

The evolution of the norm of the mean particles gravity center velocity $\left\langle u_{s}\right\rangle / u_{c}$ for the five particle volume fractions is plotted as a function of time in Fig. 8; without fluid (a) and with a fluid at a decreasing Reynolds number (b), (c) and (d). We are interested here 

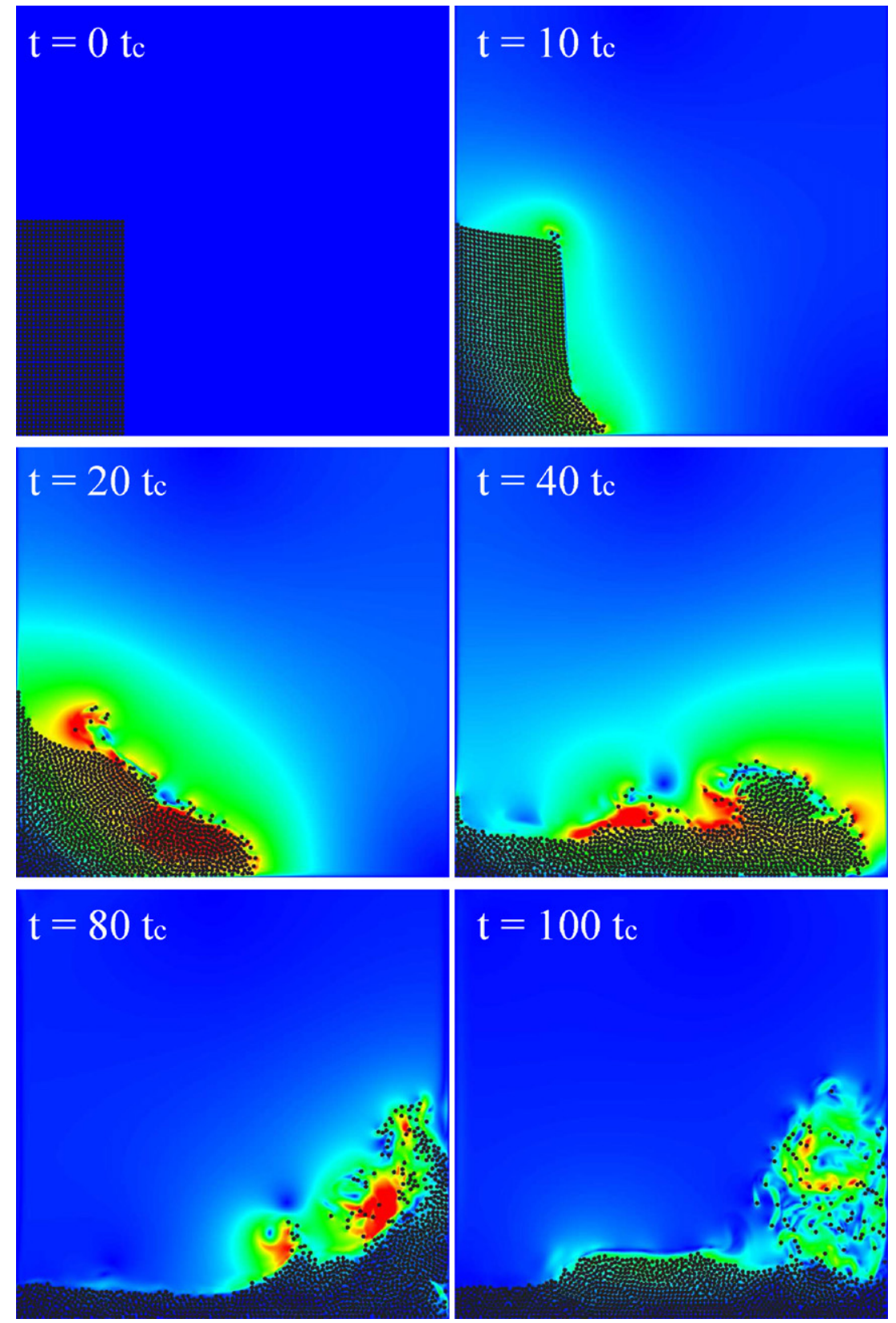

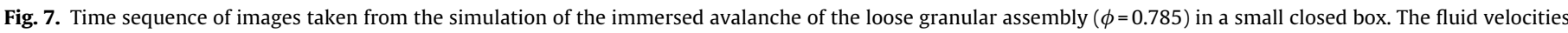
are shown in color levels. (For interpretation of the references to color in this figure legend, the reader is referred to the web version of the article.)

only in the destabilization phase, i.e., $t \in[0 ; 20] t_{c}$. We note first that without fluid (Fig. 8(a)), the initial particle volume fraction does not influence the mean velocity of the avalanche. In fact $\left\langle u_{s}\right\rangle$ increases until a maximum value, similar for all sets and reached at $t \simeq 9.0 t_{c}$, and then decreases to zero.

On the contrary, when the particles are fully immersed (see Fig. 8(b)-(d)), the avalanche is strongly affected by both the initial particle volume fraction and the Reynolds number, the maximum velocity increasing for a decreasing value of $\phi$ and $1 / R e_{c}$. As showed experimentally by Pailha et al. [38], we distinguish here two behaviors: (1) a loose behavior $(\phi=0.785)$ in which the packing destabilizes instantly; (2) a dense behavior $(\phi \geq 0.829)$ where we observe an initial relaxation time $t_{\text {relax }}$ during which $\left\langle u_{s}\right\rangle$ remains zero and whose duration depends on the granular compacity $\phi$. The value of $t_{\text {relax }}$ is reported in Fig. 9 as a function of $\phi$ for the three Reynolds numbers considered. $t_{\text {relax }}$ is obtained from velocity plots by taking the intersection between a straight fit of the acceleration phase and the time axis (a straight line is drawn in Fig. 8(d) as an illustration). It is interesting to see that $t_{\text {relax }}$ increases linearly with the initial compacity $\phi$, the Reynolds number changing slightly the slope of the linear approximation. These 2D numerical results are consistent with the experimental studies [38] suggesting that the dilatancy is responsible of the delay time before the granular assembly destabilizes. In the dense case, the dilatancy occurring when the granular assembly starts moving implies a negative pore pressure between particles which tend to stabilize the whole. In the loose case, on the contrary, the volume of interstitial pores necessarily decreases when particles collapse causing an excessive pressure that accelerates the avalanche. We note that in $2 \mathrm{D}$ the interstitial pores are not coalescent as in $3 \mathrm{D}$, implying therefore the possibility that portions of fluid were captured between particles. However, our results suggest that the mechanism is qualitatively the same in $2 \mathrm{D}$ as in 3D. 

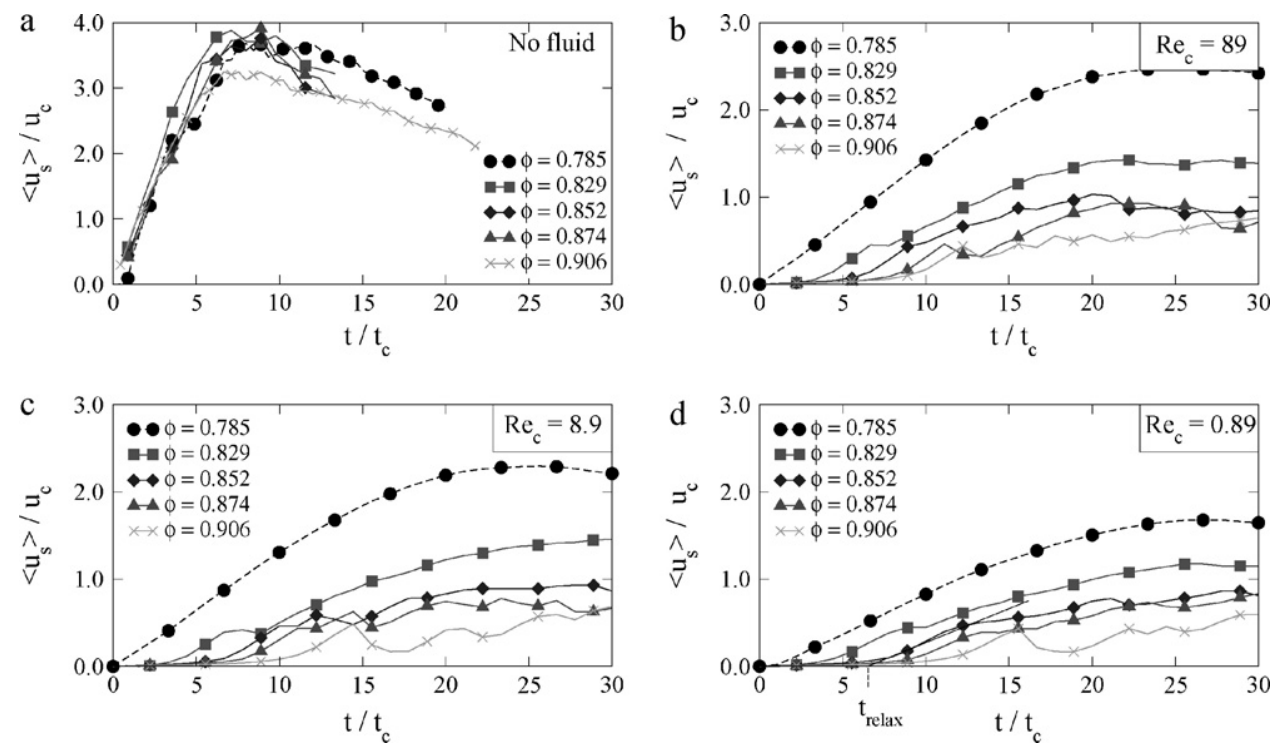

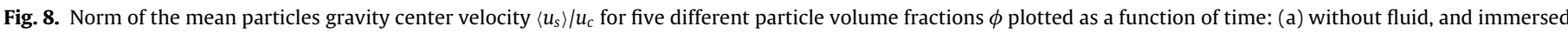
in a fluid at three different Reynolds numbers; (b) $R e_{c}=89$; (c) $R e_{c}=8.9$; (d) $R e_{c}=0.89$.

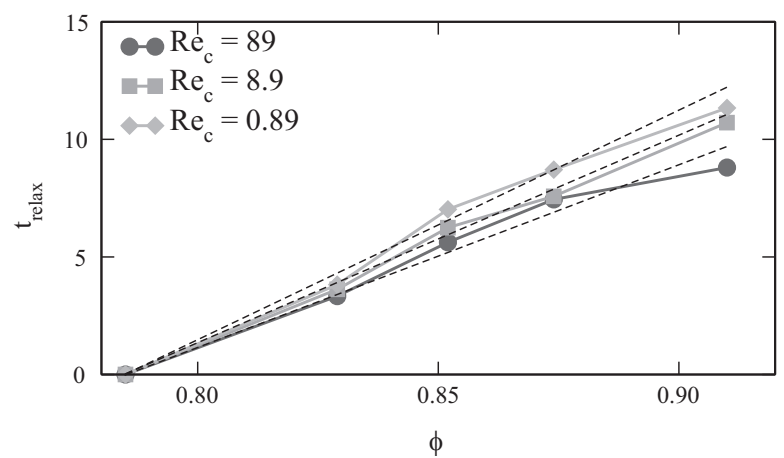

Fig. 9. Relaxation time $t_{\text {relax }}$ before the granular assembly destabilizes as a function of initial compacity $\phi$ at three different Reynolds numbers $R e_{c}$.

In order to confirm the dilatancy argument provided above, we focus on the evolution of pore volume at particle scale during the initiation phase of the avalanche. By analyzing screenshots taken at different times, we deduce the pore volume variation $\delta V / V_{0}$. From this numerical processing, we plot the evolution of $\delta V / V_{0}$ as a function of the relative horizontal displacement $\delta x / x_{0}$ for $\operatorname{Re}_{C}=89$ (see Fig. 10). A first observation is that the loosest sample $(\phi=0.785)$ presents a negative evolution of porosity whereas in the dense case $(\phi \geq 0.829)$, plots are all positive. This result is

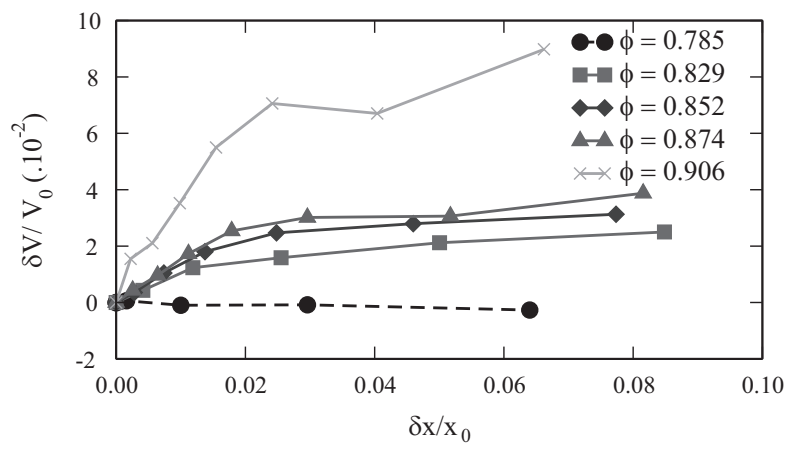

Fig. 10. Pore volume variation $\delta V / V_{0}$ as a function of the relative horizontal displacement $\delta x / x_{0}$ of the gravity center. consistent with the fact that the porosity tends to grow for dilating behaviors and reduce for contracting ones. In the dense case, we note that the porosity variation increases with $\phi$. All plots present a first regime during which the porosity quickly grows with $\delta x / x_{0}$, followed by a second regime where the porosity seems to slowly saturate towards a maximum value. In the first regime, the system dilates along a sliding direction implying locally the transition from a triangular to a rectangular configuration of the particles (in monodisperse case), and increasing at the same time the local porosity. The maximum local porosity being reached for the rectangular configuration, the particles tend naturally to come back to a triangular configuration in the second regime. Locally, an excessive pore pressure develops which accelerates the avalanche without changing significantly the global porosity.

\section{Concluding remarks}

In this work we have extended the previous micro-scale fluid/particles mixture model by replacing for the first time the MD method by a NSCD approach. The NSCD approach can be considered as an adequate framework for the numerical treatment of granular problems involving complex specific contact laws. In the frictional case, the Coulomb friction and perfectly rigid contact condition are solved in an exact form, i.e., without introducing any artificial penalization parameter or damping.

In this context, the NSCD-DLM/FD model presented in this paper has proved to be a suitable tool able to simulate complex phenomena at the macroscopic scale from a detailed description of the rheology and the texture at the particle scale. By studying how a column of particles collapses in a Newtonian fluid, we find numerically experimental results demonstrating that the dynamics of the assembly is controlled by both the local dilatancy and the interstitial pressure. We show that relatively loose granular packings seem to behave like dry assemblies whereas dense packings are dramatically affected by the presence of the fluid, the development of a negative pore pressure tending to postpone the avalanche onset. We finally focus on the evolution of the porosity as an internal variable indicating the degree of dilatancy. We confirm thus the correlation between the global dynamics (time of triggering) and the local configuration of the particles.

From a numerical viewpoint, the present work suggests that a detailed investigation of the particle-scale phenomenology is 
required to incorporate microscopic mechanisms into macroscopic models. Among the several issues that can be addressed in this scope, the question of the respective influence of static, contact and hydrodynamic forces on the rheology of dense granular flows is crucial. It will lead to a better understanding of local mechanisms such as contact lubrication or development of pressure gradients which could, by changing locally the texture, govern the transition from solid to liquid behaviors.

\section{Acknowledgments}

It is always a great pleasure to have stimulating discussions with F. Radjaï, LMGC, Montpellier, France. Thanks to R. Mozul, LMGC, Montpellier, France, for his valuable technical help.

\section{References}

[1] R. Chhabra, Bubbles, Drops and Particles in Non-Newtonian Fluids, CRC, 2007.

[2] S. Peker, S. Helvaci, Solid-liquid Two Phase Flow, Elsevier, 2007.

[3] M. Ishii, T. Hibiki, Thermo-fluid Dynamics of Two-phase Flow, Springer, 2006.

[4] R. Phillips, R. Armstrong, R. Brown, A. Graham, J. Abbott, A constitutive equation for concentrated suspensions that accounts for shear-induced particle migration, Physics of Fluids A: Fluid Dynamics 4 (1992) 30.

[5] M. Marchioro, M. Tanksley, A. Prosperetti, Flow of spatially non-uniform suspensions. Part I. Phenomenology, International Journal of Multiphase Flow 26 (2000) 783-831.

[6] D. Zhang, A. Prosperetti, Momentum and energy equations for disperse twophase flows and their closure for dilute suspensions, International Journal of Multiphase Flow 23 (1997) 425-453.

[7] F. Radjaï, S. Roux, J.J. Moreau, Contact forces in a granular packing, Chaos 9 (1999) 544-550.

[8] B. Oesterlé, Ecoulement multiphasiques: des fondements aux méthodes d'ingénierie, Hermes Sciences, 2006.

[9] C. Voivret, F. Radjai, J.-Y. Delenne, M.S. El Youssoufi, Space-filling properties of polydisperse granular media, Physical Review E 76 (2007) 021301-021312.

[10] F. Radjaï, I. Preechawuttipong, R. Peyroux, Cohesive granular texture, in: P.A. Vermeer, S. Diebels, W. Ehlers, H.J. Herrmann, S. Luding, E. Ramm (Eds.), Continuous and Discontinuous Modelling of Cohesive Frictional Materials, pp. 149-162.

[11] V. Topin, J.-Y. Delenne, F. Radjaï, L. Brendel, F. Mabille, Strength and fracture of cemented granular matter, The European Physical Journal E 23 (2007) 413-429.

[12] V. Richefeu, M.S. El Youssoufi, E. Azema, F. Radjai, Force transmission in dry and wet granular media, Powder Technology 190 (2009) 258-263.

[13] E. Azema, F. Radja, R. Peyroux, G. Saussine, Force transmission in a packing of pentagonal particles, Physical Review E 76 (2007) 011301.

[14] C.S. Peskin, The immersed boundary method, Acta Numerica (2002) 479-517.

[15] R. Glowinski, T.W. Pan, T.I. Hesla, D.D. Joseph, A distributed Lagrange multiplier/fictitious domain method for particulate flows, International Journal of Multiphase Flow 25 (1999) 755-794.

[16] A. Wachs, A DEM-DLM/FD method for direct numerical simulation of particulate flows: sedimentation of polygonal isometric particles in a Newtonian fluid with collisions, Computers \& Fluids 38 (2009) 1608-1628.
[17] A. Wachs, PeliGRIFF, a parallel DEM-DLM/FD direct numerical simulation tool for 3D particulate flows, Journal of Engineering Mathematics, in press, doi:10.1007/s10665-010-9436-2.

[18] F. Dubois, M. Jean, LMGC90 une plateforme de développement dédiée a la modélisation de problemes dínteraction, in: A.B.M. Potier-Ferry, M. Bonnet (Eds.), Sixieme Colloque National en calcul des structures, Giens, Ecole Polytechnique, pp. 111-118.

[19] J.J. Moreau, Unilateral contact and dry friction in finite freedom, in: J. Moreau, P. Panagiotopoulos (Eds.), Topics in Nonsmooth Mechanics, vol. 302, SpringerVerlag, New York, 1988, pp. 1-83.

[20] M. Jean, J.J. Moreau, Unilaterality and dry friction in the dynamics of rigid bodies collections, in: Proceedings of the Contact Mech. Int. Sym, pp. 31-48.

[21] F. Perales, S. Bourgeois, A. Chrysochoos, Y. Monerie, Two field multibody method for periodic homogenization in fracture mechanics of nonlinear heterogeneous materials, Engineering Fracture Mechanics 75 (2008) 3378-3398.

[22] F. Radjai, V. Richefeu, Contact dynamics as a nonsmooth discrete element method, Mechanics of Materials 41 (2009) 715-728.

[23] M. Jean, The non-smooth contact dynamics method, Computer Methods in Applied Mechanics and Engineering 177 (1999) 235-257.

[24] M. Jean, V. Acary, Y. Monerie, Non-smooth contact dynamics approach of cohesive materials, Philosophical Transactions of Royal Society London A 359(2001) 2497-2518.

[25] M. Iverson, M.E. Reid, N.R. Iverson, R.G. LaHusen, M. Logan, J.E. Mann, D.L. Brien, Acute sensitivity of landslide rates to initial soil porosity, Science (2000) 290.

[26] M. Iverson, The physics of debris flows, Review of Geophysics (1997) 35.

[27] M.A. Hampton, H.J. Lee, J. Locat, Submarine landslides, Review of Geophysics 34 (1996) 33.

[28] C. Cassar, M. Nicolas, O. Pouliquen, Submarine granular flows down inclined planes, Physics of Fluids 17 (2005) 103301.

[29] C. Lareo, P. Fryer, M. Barigou, The fluid mechanics of two-phase solid-liquid food flows: a review, Food and Bioproducts Processing 75 (1997) 73-105.

[30] E. Lajeunesse, J.B. Monnier, G.M. Homsy, Granular slumping on a horizontal surface, Physics of Fluids 17 (2005) 2371.

[31] P. Jop, Y. Forterre, O. Pouliquen, A constitutive law for dense granular flows Nature 441 (2006) 727-730.

[32] G. Midi, On dense granular flows, European Physical Journal E 14 (2004) 341-365.

[33] L.J.C. Lacaze, K.R.R. Phillips, Planar collapse of a granular column: experiments and discrete element simulations, Physics of Fluids 20 (2008) 1070-6631.

[34] L. Lacaze, R. Kerswell, Axisymmetric granular collapse: a transient 3D flow test of visco-plasticity, Physical Review Letter 102 (2009) 108305.

[35] F. Radjaï, D.E. Wolf, The origin of static pressure in dense granular media, Granular Matter 1 (1998) 3-8.

[36] N. Jain, J.M. Ottino, R.M. Lueptow, Effect of interstitial fluid on a granular flowing layer, Journal of Fluid Mechanics 508 (2004) 23-24

[37] D. Doppler, P. Gondret, T. Loiseleux, S. Meyer, M. Rabaud, Relaxation dynamics of water-immersed granular avalanches, Journal of Fluid Mechanics 557 (2007) $161-181$.

[38] M. Pailha, M. Nicolas, O. Pouliquen, Initiation of underwater granular avalanches: influence of initial volume fraction, Physics of Fluids (2008) 20.

[39] T. Maxworthy, J.S.J.E. Leilich, E.H. Meiburg, The propagation of a gravity current into a linearly stratified fluid, Journal of Fluid Mechanics 453 (2002) 371-394.

[40] L. Delorme, A. Colagrossi, A. Souto-Iglesias, R. Zamora-Rodrguez, E. Bota-Vera, A set of canonical problems in sloshing. Part I: Pressure field in forced rollcomparison between experimental results and SPH, Ocean Engineering 36 (2009) 168-178. 